\title{
Adenopatia supraclavicular como forma de apresentação de cancro da próstata metastizado
}

\author{
Supraclavicular lymphadenopathy as initial presentation of prostate cancer
}

\author{
Helena Temido ${ }^{1}$, Manuel Oliveira-Santos ${ }^{2}$, Francisco Parente ${ }^{1}$, Armando Carvalho ${ }^{1}$ \\ ${ }^{1}$ Serviço de Medicina Interna A do Centro Hospitalar e Universitário de Coimbra \\ ${ }^{2}$ Serviço de Cardiologia A do Centro Hospitalar e Universitário de Coimbra
}

\begin{abstract}
Resumo
A neoplasia da próstata na sua forma avançada tem geralmente metastização óssea ou para gânglios pélvicos. 0 envolvimento de gânglios supraclaviculares é raro (0,4-1\% de todos os casos) $)^{1}$ e a sua apresentação como adenopatia supraclavicular é ainda mais rara, com apenas alguns casos descritos na literatura ${ }^{2-7}$. Neste contexto, apresentamos 0 caso de um homem de 62 anos, saudável, com tumefação de novo na fossa supraclavicular esquerda, sem sintomas associados. Após a investigação estabeleceu-se 0 diagnóstico de adenocarcinoma da próstata em fase avançada, com metastização disseminada. Alertamos para uma forma rara de apresentação de adenocarcinoma da próstata e discutimos a abordagem diagnóstica em casos de adenopatias isoladas.

Palavras-chave: supraclavicular, próstata, cancro, metastização, apresentação
\end{abstract}

\section{Introdução}

Em doentes que se apresentem com adenopatia supraclavicular isolada é necessário fazer o diagnóstico diferencial com várias patologias, nomeadamente com neoplasias. No entanto, o adenocarcinoma da próstata não é uma das hipóteses inicialmente consideradas. Este é diagnosticado frequentemente por elevação assintomática dos valores de Prostate Specific Antigen (PSA) ou por investigação de sintomas de invasão local e metastização ganglionar pélvica ou metastização à distância (pulmonar e óssea) já com sintomas sistémicos. Os cancros que mais frequentemente se apresentam com adenopatias supraclaviculares são cancros da nasofaringe, orofaringe e trato gastrointestinal superior. 0 envolvimento de gânglios supraclaviculares pela neoplasia da próstata acontece em apenas $0,4-1 \%$ dos $\operatorname{casos}^{1}$ e a sua apresentação como adenopatia supraclavicular é ainda mais invulgar ${ }^{2-7}$.

Ainda que extremamente rara, esta pode ser a forma de apresentação de adenocarcinoma prostático metastizado, como descrevemos no caso clínico que se segue.

\section{Caso clínico}

Doente do sexo masculino, 62 anos, que recorreu ao Serviço de Urgência por apresentar uma tumefação indolor na fossa supraclavicular esquerda, não sabendo referir o tempo de evolução. Negava traumatismo local, sintomas constitucionais, queixas respiratórias e alterações do trânsito gastrointestinal ou génito-urinário. Não tinha antecedentes pessoais patológicos ou contexto epidemiológico de relevo. Não fazia medicação crónica. Ao exame

\begin{abstract}
Prostate cancer in its advanced form usually metastasizes to the bone or pelvic ganglia. The involvement of the supraclavicular lymph nodes is rare ( $0.4 \%$ to $1 \%$ of all cases) $)^{1}$. Its presentation as a supraclavicular lymphadenopathy is even more unusual with only a few cases described in the literature ${ }^{2-7}$. In this context, we describe the case of a 62 year old man presenting with a left supraclavicular mass without any other signs or associated symptoms. After all the workup, we established the diagnosis of widely metastasized prostate adenocarcinoma. We bring forward this case as an example of a rare form of presentation of prostate cancer and we use it as a base for discussing an approach to isolated peripheral lymphadenopathy. Keywords: supraclavicular, prostate, cancer, metastasis, presentation
\end{abstract}

físico apresentava bom estado geral, sem evidência de icterícia ou anemia. Era evidente uma tumefação duro-elástica, móvel e indoIor na região supraclavicular esquerda. Sem outras adenopatias palpáveis. Não apresentava alterações ao exame objetivo do tórax. À palpação abdominal não eram objetiváveis massas ou organomegálias. 0 toque rectal que revelava uma próstata de tamanho normal mas dura e fixa. Não apresentava linfedema dos membros inferiores ou sinais de trombose venosa periférica. 0 exame neurológico sumário não demonstrava alterações do estado mental, alterações da coordenação e marcha, alterações da sensibilidade, força ou reflexos. Analiticamente sem alterações de hemograma, eletroforese de proteínas séricas, transaminases, fosfatase alcalina, gamaGT, proteína $\mathrm{C}$ reativa. As serologias e marcadores tumorais eram negativos, excetuando-se o PSA que era elevado (81ng/ml). A ecografia da tumefação supraclavicular revelava um conglomerado adenopático (Figura 1).

Com o diagnóstico de adenocarcinoma da próstata com metastização disseminada, o doente iniciou terapêutica com Ciproterona e Triptorrelina com diminuição inicial dos valores de PSA. Posteriormente veio a desenvolver sintomas de obstrução do trato urinário e após 12 meses em terapêutica de castração hormonal verificou-se agravamento clínico e subida dos valores de PSA. Iniciou terapêutica com Abiraterona, sem resultado, acabando por falecer 18 meses depois do diagnóstico.

\section{Discussão}

A neoplasia da próstata é a segunda maior causa de morte por cancro em homens nos países desenvolvidos ${ }^{8}$. 0 diagnóstico pode ser feito por elevação assintomática do PSA ou por investigação de sintomas relacionados com invasão local da neoplasia e metastização ganglionar pélvica ou 
Figura 1. Ecografia de tecidos moles da fossa supraclavicular esquerda revelando nódulo linfático.

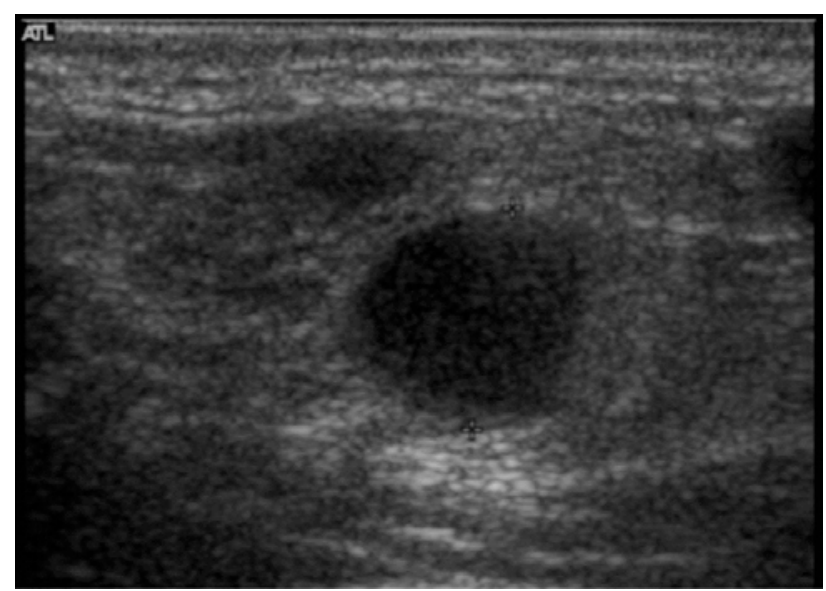

Figura 2. Histologia de linfonodo com coloração com PSA (castanho-avermelhado).

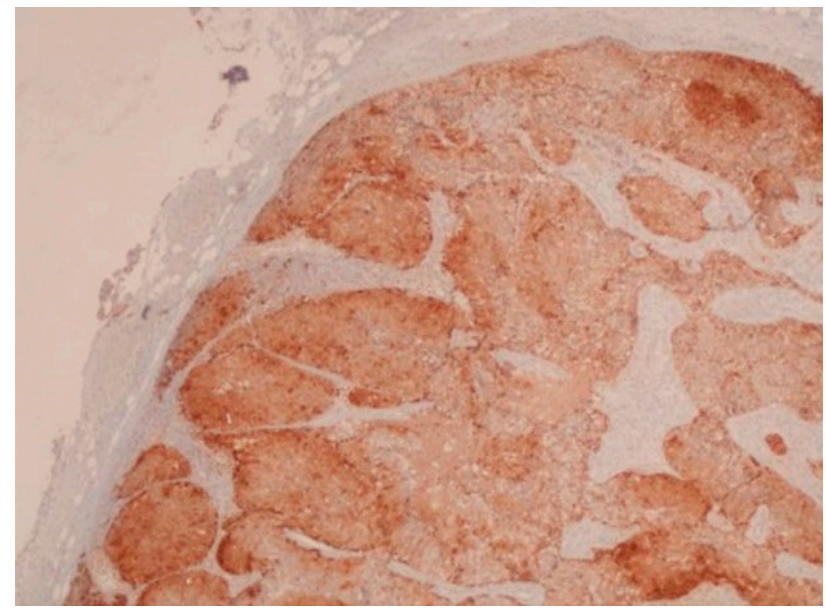

Figura 3. TAC toracoabdominopélvica com nódulos hepáticos compatíveis com lesões metastáticas.

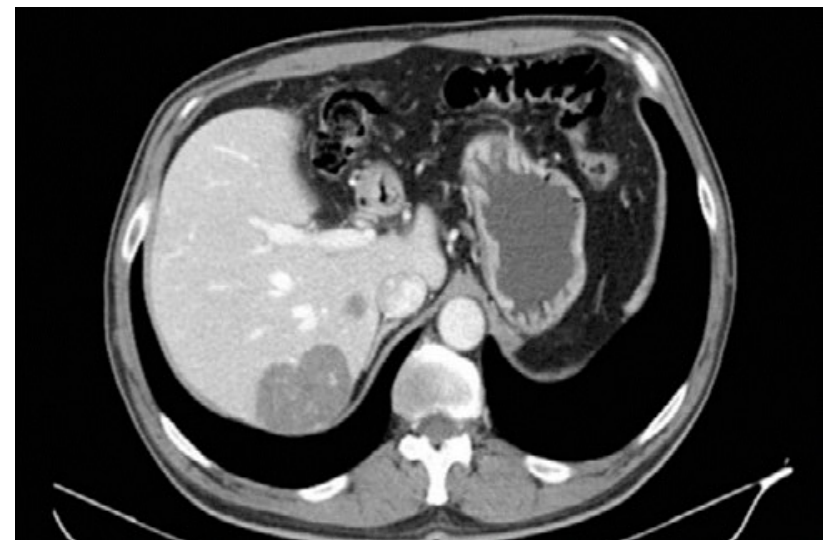

Figura 4. Cintigrafia do esqueleto revelando metastização óssea disseminada.
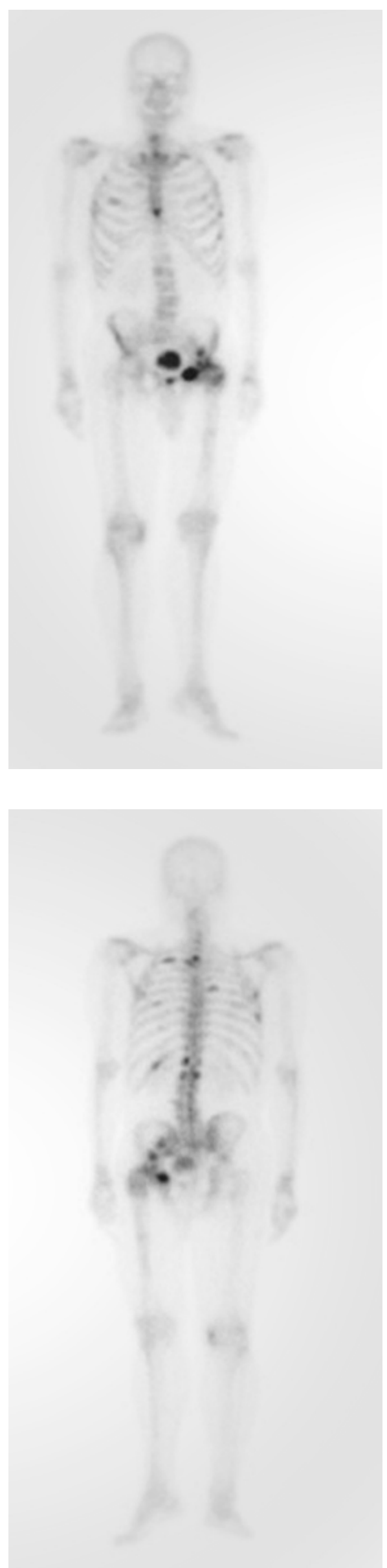
metastização à distância (pulmonar e óssea) com sintomas sistémicos. Quando metastizada, a neoplasia prostática dissemina-se para gânglios linfáticos pélvicos ou do retroperitoneu, posteriormente para 0 osso, pulmão, bexiga, fígado e glândulas supra-renais. A metastização da neoplasia da próstata para gânglios supraclaviculares acontece em apenas 0.4 a 1\% dos casos e a sua apresentação como adenopatia supraclavicular é ainda mais invulgar ${ }^{2-7}$.

Dada a raridade desta forma de apresentação de adenocarcinoma da próstata é necessário um elevado grau de suspeição para fazer este diagnóstico quando estamos perante um doente que se apresenta com adenopatia supraclavicular isolada. Nestes casos, o diagnóstico diferencial deve incluir várias patologias, nomeadamente infecções e neoplasias. Deve ser colhida uma história clínica completa, incluindo sinais ou sintomas associados, contexto epidemiológico e viagens recentes, hábitos tabágicos e sexuais, medicação crónica e história de neoplasias. 0 exame físico também deve ser completo, incluindo pesquisa de outras adenopatias, sua localização e avaliação de sinais de risco para malignidade como o seu tamanho, consistência e mobilidade. As adenopatias localizadas na fossa supraclavicular têm maior probabilidade de serem malignas e devem ser sempre investigadas, nomeadamente com biópsia. Realça-se também a pertinência da realização do toque rectal no exame físico inicial destes doentes e o pedido do doseamento do PSA no estudo analítico. As alterações encontradas podem trazer pistas importantes para acelerar a marcha diagnóstica. Na literatura, Butler et al $(n=19)^{9}$ descrevem que 58\% dos doentes com adenopatia supraclavicular isolada tinham toque rectal normal e Saeter et al $(n=24)^{10}$ descrevem que $20 \%$ dos doentes tinham valores normais de PSA no sangue. Assim, a realização da coloração específica para PSA nas amostras de biópsia da adenopatia supraclavicular é fundamental para o diagnóstico.

Devemos ainda salientar que o diagnóstico atempado de neoplasia da próstata metastizada é essencial na escolha de tratamento, uma vez que terapêutica de bloqueio hormonal mais intensa ab initio (tal como a utilizada em doentes com metastização óssea ou pulmonar) se traduz num melhor prognóstico para o doente.

Com a apresentação deste caso clínico, pretendemos destacar a importância de considerar a neoplasia da próstata no diagnóstico diferencial de doentes do sexo masculino que se apresentem com adenopatia supraclavicular isolada. Pretendemos ainda relembrar que na abordagem inicial destes doentes deve ser feito o toque rectal, pedido o doseamento do PSA e pedida a coloração específica para PSA na análise histológica da peça de biópsia.

\section{Referências bibliográficas}

1. Davarci M, Gokce A, Guven EO, Yalcinkaya FR, Esen H, Sevinc A. Metastatic prostate adenocarcinoma presenting as supraclavicular lymphadenopathy: a report of two cases. ContempOncol (Pozn). 2012;16(1):53-55.

2. Zhu L, Luo C, Wu W, Ying J, Zhong H. Prostate adenocarcinoma with negative immunohistochemical stain of prostate-specific antigen presenting with cervical mass: A case report. Journal of Research in Medical Sciences : The Official Journal of Isfahan University of Medical Sciences. 2013;18(9):814-817.

3. Bhattar R, Maheshwari A, Yadav SS, Tomar V. Unusual Presentation of Prostate Carcinoma: A Case Report. Journal of Clinical and Diagnostic Research : JCDR. 2017;11(2):PD06-PD07.

4. Sepúlveda L, Gorgal T, Pires V, Rodrigues F. Prostate Cancer Metastatic to the Cervical Lymph Nodes. Case Reports in Urology, vol2015; Article ID 263978, 4 pages, 2015.

5. Lad M Sharma A Patten Darren. A surprising diagnosis: metastatic prostate cancer causing cervical lymphadenopathy. BMJ Case Rep. 2014; 2014: bcr2013201630

6. Ahamed S, Agarwal A, Raju P. Metastatic Prostate Carcinoma Presenting as Supraclavicular Lymphadenopathy - Is it Unusual? Annals of The Royal College of Surgeons of England. 2006;88(6):W4-W5.

7. Hematpour K, Bennett CJ, Rogers D, Head CS, Supraclavicular lymph node: Incidence of unsuspected metastatic prostate cancer. Eur Arch Otorhinolaryngol. 2006 Sep: 263 (9): 872-4.

8. United States Cancer Statistics: 1999-2013 Incidence and Mortality Web-based Report. Atlanta (GA): Department of Health and Human Services, Centre for Disease Control and Prevention and National Cancer Institute. 2016.

9. Butler $\mathrm{J}$ et al. Enlargement of the supraclavicular lymph nodes as the initial sign of prostatic carcinoma. Cancer. 1971 May;27(5):1055-63.

10. Saeter $G$ et al. Carcinoma of the Prostate with Soft Non-regional Lymphatic Metastases Diagnosis: a Review of 47 Cases. British Journal of Urology, (1984). 56, 385-390. 